\title{
Recursive Preferences and Balanced Growth ${ }^{1}$
}

\author{
Roger E. A. Farmer ${ }^{2}$ \\ UCLA, and CEPR \\ rfarmer@econ.ucla.edu
}

\author{
Amartya Lahiri \\ Federal Reserve Bank of New York \\ amartya.lahiri@ny.frb.org
}

This Draft: July 2004

${ }^{1}$ Both authors acknowledge the support of UCLA academic senate grants. We thank Robert Becker, Mick Devereux, Martin Schneider and Ping Wang for helpful feedback. We also thank two referees for detailed comments that have substantially improved the final version of this paper.

${ }^{2}$ Corresponding Address: Roger E. A. Farmer, Department of Economics, 8283 Bunche Hall, UCLA, Los Angeles, CA 90095-1477; Telephone: (310) 825-6547; Fax: (310) 825-9528. 


\begin{abstract}
We study a class of utility functions that are defined recursively by an aggregator $W(x, y)$ where $u_{t}=W\left(c_{t}, u_{t+1}\right)$. In single-agent economies it is known that a sufficient condition for the existence of a balanced growth path is that utility should be homogenous of degree $\gamma$. In the context of a multi-agent economy we show that this restriction implies that either a balanced growth equilibrium fails to exist or all agents have the same constant discount factor. We suggest a generalization of recursive preferences wherein the intertemporal utility function is time dependent. Within this class we establish that there may exist a balanced growth equilibrium even if agents are different.
\end{abstract}




\section{Introduction}

Two central features of most modern macroeconomic models are (a) the assumption of infinitely lived families with intertemporally separable utility functions; and (b) an environment that allows for balanced growth. While the first stems primarily from a desire for simplicity, the balanced growth construct originates in the seminal work of Kaldor [8] who stressed that balanced growth provides a good characterization of the long run development experience of the currently industrialized countries. According to the Kaldor growth facts, development paths are characterized by the constancy of growth rates, factor income shares and capital-output ratios. From a modelling standpoint, balanced growth is an attractive feature since it makes otherwise complicated environments simple to analyze.

It is well known that the assumption of time separability of preferences is restrictive since, as conjectured by Ramsey [13] and established by Becker [1], all agents must have the same rate of time preference if a model in this class is to display a non-degenerate wealth distribution. If rates of time preference are different across agents then the most patient family will asymptotically own all of world wealth. ${ }^{1}$ A number of authors, starting with Koopmans [10] and Uzawa [16], have studied recursive preferences in which the rate of time preference is a function of future consumption sequences and Lucas-Stokey [11] and Epstein-Hynes [7] have used these preferences to construct examples of economies for which there exists a non-degenerate asymptotic wealth distribution. ${ }^{2}$

In the Lucas and Stokey [11] environment, agents have recursive preferences defined over bounded consumption sequences. Within this environment they proved the existence of a nondegenerate income distribution; but their result does not apply to economies with growth. Boyd [4] generalized recursive utility to a class of preferences defined over unbounded consumption sequences and his work does permit the study of environments that permit growth. Using Boyd's results, Dolmas [6] proved that there exists a balanced growth path in the representative agent growth model if there is a recursive representation of preferences, $u_{t}=W\left(c_{t}, u_{t+1}\right)$ for which the

\footnotetext{
${ }^{1} \mathrm{~A}$ comprehensive discussion of these and related results is contained in Becker and Boyd [2].

${ }^{2}$ See, for example, Becker and Boyd [2], Boyd [4], Dolmas [6], Epstein and Hynes [7], and Lucas and Stokey [11].
} 
aggregator $W(x, y)$ is homogeneous of the form $W\left(\lambda x, \lambda^{\gamma} y\right)=\lambda^{\gamma} W(x, y)$.

In this paper we study the conditions under which recursive preferences, balanced growth, and heterogenous preferences (i.e., multiple agents) can coexist. When preferences are described by homothetic utility functions, (a necessary condition for the existence of balanced growth) we show that the asymptotic wealth distribution in a multi-agent economy is degenerate or it reflects the initial allocation of wealth. This result implies that all types of non-trivial wealth distributions under balanced growth are already realized by additive preferences. The result is troubling given that a key motivation for introducing recursive preferences is to permit heterogeneity without inducing a degenerate wealth distribution.

To recover the property of non-degeneracy of the wealth distribution we introduce an exogenous time-dependence into the utility function and show that this formulation permits the coexistence of recursive preferences, preference heterogeneity, and balanced growth. We suggest an application of time dependence in which agents care about their relative position in the world wealth distribution; in this context world wealth enters utility as an external effect. Using this example, we show that our formulation is consistent with a determinate distribution of world wealth.

\section{Preliminaries}

Our approach follows Lucas and Stokey [11] as adapted by Boyd [4]. Let

$$
\mathbf{X}(g)=\left\{\left.X|| X\right|_{\mathbf{g}}<\infty, x_{t} \geq 0\right\}
$$

be the space of feasible consumption sequences where $|X|_{g}=\sup \left|x_{t} / g^{t-1}\right|$ for $g \geq 1$ is the $g$-weighted $l^{\infty}$ norm ${ }^{3}$. In words, a consumption sequence is feasible if the sequence $\left\{c_{t} / g^{t-1}\right\}$ is bounded. The following, due to Boyd [4], defines a metric on the space of continuous functions $f: \mathbf{X}(g) \rightarrow R$.

\footnotetext{
${ }^{3}$ We use the convention that uppercase letters represent vectors in $R^{\infty}$, lowercase letters are scalars and boldface letters represent sets.
} 
Definition 1 (Boyd) Let $f \in c(\mathbf{A}, \mathbf{B})$, where $c(\mathbf{A}, \mathbf{B})$ is the space of continuous functions from $\mathbf{A}$ to $\mathbf{B}$. Suppose $\varphi \in c(\mathbf{A}, \mathbf{B})$ with $\mathbf{B} \subset R$ and $\varphi>0$. The function $f$ is $\varphi$-bounded if the $\varphi$-norm of $f,\|f\|_{\varphi}=\sup \{|f(x)| / \varphi(x)\}$ is finite. The $\varphi$-norm turns $c_{\varphi}(\mathbf{A}, \mathbf{B})=\{f \in c(\mathbf{A}, \mathbf{B}): f$ is $\varphi$-bounded $\}$ into a complete metric space.

Next, we introduce a class of aggregators $W: \mathbf{X}(g) \times \mathbf{Y} \rightarrow \mathbf{Y} . W(x, y)$ maps period $t$ consumption, $(x \in X(g))$, and period $t+1$ utility, $(y \in R)$, into period $t$ utility. We assume that $W$ satisfies the following properties.

\section{Assumption $U 1 \quad W(0,0)=0$}

Assumption $U 2 \quad W$ is continuous, and increasing in both arguments

Assumption $U 3 \quad 0 \leq W(x, 0) \leq A\left(1+x^{\eta}\right), \quad A, \eta>0$

Assumption $U 4 \quad W$ is differentiable and $\varphi$-bounded for $\varphi(X)=1+|X|_{g}^{\eta}$

Assumption $U 5 \quad\left|W(x, y)-W\left(x, y^{\prime}\right)\right| \leq \delta\left|y-y^{\prime}\right|$ for all $x \in \mathbf{X}(g), y, y^{\prime} \in \mathbf{Y}$ where $\delta g^{\eta}<1$

Assumption $U 6 \quad\left(T_{W}^{N} y\right)(X)$ is concave in $X$ for all $N$ and all constants $y \in \mathbf{Y}$.

Assumptions $U 2$ and $U 5$ correspond to $W 1$ and $W 2$ in Boyd ([4] page 330). Assumption $U 1$ restricts us to aggregators that are bounded below and $U 3$ allows us to define a natural concept of distance using the definition of $\varphi$-boundedness. Specifically, this assumption allows us to define a function $\varphi$ :

$$
\varphi(X)=1+|X|_{g}^{\eta}
$$

where $|X|_{g}$ is the $g$-weighted $l^{\infty}$ norm.

Given assumptions $U 1-U 5$ it follows from Boyd's Continuous Existence Theorem ([4] page 333) that there exists a unique $u \in c_{\varphi}$ such that

$$
W(\pi X, u(S X))=u(X)
$$

where $\pi X=x_{1}$, is the projection operator and $S X=\left(x_{2}, x_{3}, \ldots\right)$ is the shift operator. Moreover, from Boyd's Lemma 1 ([4] page 331), assumption U6 guarantees the concavity of $u$.

We now turn our attention to finding a suitable upper bound on the rate at which consumption sequences are permitted to grow. We study an economy where the net output of the economy is 
characterized by a function $f\left(k_{t}, g^{t}\right)$ where $g>1$ represents the growth factor of technological progress. At date $t$ we assume that output is divided between consumption $c_{t}$ and next period's capital stock $k_{t+1}$

$$
c_{t}+k_{t+1}=f\left(k_{t}, g^{t}\right), \quad t=1,2 \ldots
$$

We assume that $f$ satisfies the following properties;

Assumption $P 1 \quad f$ is continuous

Assumption $P 2 \quad f$ is concave

Assumption P3 $f$ is twice continuously differentiable

Assumption P4 $f$ is linearly homogeneous

Assumption P5 $\quad \lim _{k \rightarrow \infty} f_{k}(k, a)=0$

Assumption $P 6 \quad \lim _{k \rightarrow 0} f_{k}(k, a)=\infty$

Define $f^{t}(k)$ inductively by the initial condition $f^{1}=f(k, 1)$ and the recursion $f^{t}(k)=f\left(f^{t-1}(k), g^{t-1}\right)$. The path of pure accumulation, defined as $\left\{f^{t}(k)\right\}_{t=1}^{\infty}$, is the sequence of capital stocks that would be attained if all of society's resources were invested in every period.

Let $x_{t}=k_{t} / g^{t}$. Then for the class of constant returns to scale neoclassical production functions that satisfy the Inada conditions $P 5$ and $P 6$, the map $g x_{t+1}=f\left(x_{t}, 1\right)$ has two fixed points, $\bar{x}=0$ and $\bar{x}=f(\bar{x}, 1) / g$. Assumptions $P 1-P 6$ also imply that $f^{t}(k) / g^{t}$ converges to $f(\bar{x}, 1) / g$ for all $k \geq 0$. For this class of functions,

$$
\lim _{t \rightarrow \infty}\left[\frac{f^{t}(k)}{g^{t}}=\bar{k}<\infty\right]
$$

and there is no loss to restricting feasible consumption and capital sequences to lie in $\mathbf{X}(g)$, the set of sequences in $R^{\infty}$ for which $x_{t} / g^{t}$ is bounded.

\section{Implications of Balanced Growth}

In this section we study the solution to the problem faced by a benevolent social planner. We assume that the social planner maximizes the welfare of a representative agent subject to the constraints 
imposed by a technology that satisfies properties $P 1-P 6$ and the sequence of resource constraints defined by Equation (1) for each date $t=1, \ldots$. Within this framework we ask: What restrictions must be placed on preferences and technology for the solution to a Social Planner's problem to be consistent with balanced growth? We define balanced growth as follows.

Definition 2 A balanced growth path is a set of sequences $\{K, C, Y\}$ for which there exists a triple $\{k, c, y\}$ such that if $k_{0}=k$ then

$$
k_{t}=k g^{t}, \quad c_{t}=c g^{t}, \quad y_{t}=f\left(k_{t}, g^{t}\right)=y g^{t} .
$$

A social planning optimum dictates that the marginal rate of transformation, $f_{k}\left(k_{t}, g^{t}\right)$ must equal the marginal rate of substitution (MRS) for every date $t$;

$$
M R T \equiv f_{k}\left(k_{t}, g^{t}\right)=\frac{W_{u}\left(c_{t}, u_{t+1}\right) W_{c}\left(c_{t+1}, u_{t+2}\right)}{W_{c}\left(c_{t}, u_{t+1}\right)}=\frac{\partial u / \partial c_{t+1}}{\partial u / \partial c_{t}} \equiv M R S .
$$

Along a balanced growth path $f_{k}$ is constant since $f$ is linearly homogeneous. It follows that the MRS must also be constant for the solution to the planning problem to be consistent with balanced growth. King Plosser and Rebelo [9] showed, for the case of additively-time-separable preferences, that the MRS is constant if and only if utility is homogeneous of some degree $\gamma$ in consumption. The following lemma extends the results of King-Plosser-Rebelo to the case of a recursive utility function.

Lemma 1 For a utility function $u(C)$ to display a constant marginal rate of substitution along all balanced growth paths it is necessary and sufficient that the function $u(C)$ is homothetic. ${ }^{4}$

Homotheticity is restrictive but necessary if we are to construct models in which the equilibria are consistent with balanced growth. A subclass of homothetic functions is the class of timeseparable homogenous utility functions studied by King-Plosser-Rebelo [9]. A larger subclass is that of homogenous functions that are not necessarily separable. The following theorem, due to Dolmas, shows how to construct such functions using a class of recursive aggregators that display a certain homogeneity property. It also establishes the connection between aggregators of this class and the marginal rate of substitution along balanced growth paths.

\footnotetext{
${ }^{4}$ The proof of Lemma 1 is straightforward and is omitted in the interests of brevity.
} 
Theorem 1 (Dolmas) Suppose $W$ satisfies assumptions $U 1-U 5$ and is such that $W\left(\lambda x, \lambda^{\gamma} y\right)=$ $\lambda^{\gamma} W(x, y)$ for all $x$ and $y$ and all $\lambda>0$, for some $\gamma$. Then, the recursive utility function $u$ exists and is homogeneous of degree $\gamma$. If $W$ is also once-differentiable, the marginal rate of substitution exists and is a constant along a balanced growth path .

Homogenous aggregators of this type have some useful properties that were noted by Dolmas [6]. In particular, if the utility function is constructed from a recursive aggregator and $u(C)$ is homogeneous of degree $\gamma$, then the aggregator $W\left(c_{t}, u_{t+1}\right)$ satisfies the conditions

$$
\begin{gathered}
W\left(\lambda c_{t}, \lambda^{\gamma} u_{t+1}\right)=\lambda^{\gamma} W\left(c_{t}, u_{t+1}\right), \\
W_{c}\left(\lambda c_{t}, \lambda^{\gamma} u_{t+1}\right)=\lambda^{\gamma-1} W_{c}\left(c_{t}, u_{t+1}\right), \\
W_{u}\left(\lambda c_{t}, \lambda^{\gamma} u_{t+1}\right)=W_{u}\left(c_{t}, u_{t+1}\right) .
\end{gathered}
$$

Equation (3) implies that, along any balanced growth path, utilities in adjacent periods are related by the expression, $u_{t+1}=g^{\gamma} u_{t}$.

Lucas and Stokey [11] studied a multi-agent economy without growth in which agents have recursive preferences. They provided a sufficient condition for the existence of a non-degenerate asymptotic wealth distribution that is independent of the initial endowment. In their framework each individual's discount factor is endogenously determined by his consumption allocation. The following theorem illustrates why this mechanism breaks down if utility functions are homogeneous. Theorem 2 Suppose $W$ satisfies assumptions $U 1-U 5$ and is such that $W\left(\lambda x, \lambda^{\gamma} y\right)=\lambda^{\gamma} W(x, y)$ for all $x$ and $y$ and all $\lambda>0$, for some $\gamma$. Suppose also that $W$ is differentiable: then along a balanced growth path, the marginal rate of substitution can be written as

$$
\operatorname{MRS}(C, g)=g^{\gamma-1} \beta(g)
$$

where $\beta(g)$ is an endogenous discount factor. $\beta(g)$ depends on the growth rate of consumption, $g$, but is independent of its initial level, $c$. 
Proof. The MRS is defined as

$$
M R S=\frac{W_{u}\left(c_{t}, u_{t+1}\right) W_{c}\left(c_{t+1}, u_{t+2}\right)}{W_{c}\left(c_{t}, u_{t+1}\right)}
$$

Define the function $\phi(c)$ as

$$
\phi(c)=W\left(c, g^{\gamma} \phi(c)\right)
$$

$\phi(c)$ is the utility associated with the balanced growth consumption sequence $\left\{c g^{t}\right\}$. Using homogeneity properties (3-5) Equation (6) can be simplified as follows;

$$
\operatorname{MRS}(C, g)=g^{\gamma-1} \beta(c, g)
$$

where

$$
\beta(c, g) \equiv W_{u}\left(c, g^{\gamma} \phi(c)\right)
$$

is a recursive generalization of the concept of a discount factor. To establish the claim of Theorem 2 we must show that

$$
\beta(c, g) \equiv W_{u}\left(c, g^{\gamma} \phi(c)\right)=W_{u}\left(c^{\prime}, g^{\gamma} \phi\left(c^{\prime}\right)\right) \equiv \beta(g)
$$

for all $c, c^{\prime} \in R_{+}$. Let $\lambda=c / c^{\prime}$ and write

$$
W_{u}\left(c, g^{\gamma} \phi(c)\right)=W_{u}\left(\lambda c^{\prime}, g^{\gamma} \phi\left(\lambda c^{\prime}\right)\right)
$$

From homogeneity of $W_{u}$ (Equation (5)) we have that

$$
W_{u}\left(\lambda c^{\prime}, g^{\gamma} \phi\left(\lambda c^{\prime}\right)\right)=W_{u}\left(c^{\prime}, \frac{g^{\gamma} \phi\left(\lambda c^{\prime}\right)}{\lambda^{\gamma}}\right)
$$

hence

$$
W_{u}\left(c, g^{\gamma} \phi(c)\right)=W_{u}\left(c^{\prime}, \frac{g^{\gamma} \phi\left(\lambda c^{\prime}\right)}{\lambda^{\gamma}}\right) .
$$

By homogeneity of $W$, (Equation (3))

$$
\phi(\lambda c)=\lambda^{\gamma} \phi(c)
$$

hence

$$
\frac{\phi\left(\lambda c^{\prime}\right)}{\lambda^{\gamma}}=\phi\left(c^{\prime}\right)
$$


Now replacing $\phi\left(\lambda c^{\prime}\right) / \lambda^{\gamma}$ by $\phi\left(c^{\prime}\right)$ in (7) gives

$$
W_{u}\left(c, g^{\gamma} \phi(c)\right)=W_{u}\left(c^{\prime}, g^{\gamma} \phi\left(c^{\prime}\right)\right) \equiv \beta(g)
$$

Lucas and Stokey [11] study economies without growth. In such economies the discount factors $\beta_{j}$ for the $n$ agents indexed by $j=1, . . n$ are functions of their consumption allocations. These discount factors are endogenously equalized through variations in individual-specific consumption. Theorem 2 states that if preferences are homogenous (a necessary condition for balanced growth), the discount factor of an agent depends on the growth rate of consumption but not on its level. Since $g$ is the same for all agents on a balanced growth path, the Lucas-Stokey mechanism breaks down. This theorem is related to a result derived by Ben-Gad [3] who studied competitive equilibria in a multi-agent Cass-Koopmans economy in which agents have recursive preferences. He showed that, if preferences are in the class considered in our Theorem 1, then the steady state wealth distribution will be indeterminate. Our Theorem 2 is a direct implication of Ben-Gad's main result.

In economies with multiple agents, the wealth distribution will be non-degenerate only if each agent has the same marginal rate of substitution. Theorem 2 implies that the assumption of recursivity adds nothing of interest to the study of the wealth distribution beyond the case of additively separable preferences. An $n$-agent economy populated by agents with recursive homogeneous preferences has a non-trivial wealth distribution only if the marginal rates of substitution are related by the equation

$$
g^{\gamma_{i}-1} \beta_{i}(g)=g^{\gamma_{j}-1} \beta_{j}(g), \quad \text { for all } \quad i, j=1, \ldots n
$$

Although it is possible to construct examples of economies in which this condition holds, the asymptotic wealth distribution in an economy of this class would not be endogenous in the sense of Lucas-Stokey. Just as in the case of additively separable preferences, the distribution of wealth in an economy of this kind would be determined by initial conditions. Further, if one were to construct a nontrivial example in which there was a non-degenerate wealth distribution when technological progress occurred at some exogenous rate $g$, this distribution would not be robust to a small change 
in the growth rate since, unless all agents are identical, the MRS of each agent is a non-trivial function of $g$.

\section{Time Dependent Preferences}

In this section we suggest a way of endogenizing the wealth distribution in a growing economy by introducing an exogenous time-dependent factor into preferences In Section 6 we construct a model in which households care about their consumption relative to an exogenously growing reference bundle. One possible interpretation of our economy is that agents care about their relative position in the world income distribution.

We begin by expanding the commodity space. Consider aggregator functions $W^{i}: \mathbf{X}(g)^{2} \times R \rightarrow R$ and let $W^{i}$ satisfy properties $U 1-U 6$ where $W(x, y)$ refers to a function in which $x$ has two elements; the first represents consumption and the second represents an exogenous sequence $A=\left\{a_{1}, a_{2}, a_{3} \ldots\right\}$ where $a_{t}=g^{t}$. The existence theorems of Boyd and Dolmas do not restrict $c$ to be a scalar, hence one can appeal to these theorems to assert that there is a unique well defined solution $u^{i}\left(C^{i}, A\right)$ to the functional equation

$$
u^{i}\left(C^{i}, A\right)=W^{i}\left(\pi C^{i}, \pi A, u^{i}\left(S C^{i}, S A\right)\right)
$$

where $\pi A=a_{1}, S A=\left(a_{2}, a_{3}, \ldots\right)$. By including the exogenous sequence $A$ in preferences, we are able to maintain consistency with balanced growth and allow discount factors to vary along a balanced growth path.

The homogeneity properties (3-5) have the following analogs in the economy where utility depends on time;

$$
\begin{gathered}
W^{i}\left(\lambda c_{t}^{i}, \lambda a_{t}, \lambda^{\gamma_{i}} u_{t+1}^{i}\right)=\lambda^{\gamma_{i}} W\left(c_{t}^{i}, a_{t}, u_{t+1}^{i}\right), \\
W_{c}^{i}\left(\lambda c_{t}^{i}, \lambda a_{t}, \lambda^{\gamma_{i}} u_{t+1}^{i}\right)=\lambda^{\gamma_{i}-1} W_{c}\left(c_{t}^{i}, a_{t}, u_{t+1}^{i}\right), \\
W_{u}^{i}\left(\lambda c_{t}^{i}, \lambda a_{t}, \lambda^{\gamma_{i}} u_{t+1}^{i}\right)=W_{u}^{i}\left(c_{t}^{i}, a_{t}, u_{t+1}^{i}\right) .
\end{gathered}
$$


If we define the growth weighted variables

$$
\begin{gathered}
\tilde{x}_{t}=\frac{x_{t}}{g^{t}}, \quad x=\left\{c^{1}, c^{2}, y, k\right\}, \\
\tilde{u}_{t}^{1}=\frac{u_{t}^{1}}{g^{\gamma_{1} t}}, \quad \tilde{u}_{t}^{2}=\frac{u_{t}^{2}}{g^{\gamma_{2} t}},
\end{gathered}
$$

we can express the rate of time preference along a balanced growth path by the function

$$
\beta\left(\tilde{c}^{i}, g\right)=W_{u}^{i}\left(\tilde{c}^{i}, 1, g^{\gamma_{i}} \phi^{i}\left(\tilde{c}^{i}\right)\right)
$$

The terms $\tilde{c}^{i}$ and $\tilde{u}^{i}$ are related to each other by the functions $\phi^{i}(\tilde{c}), i=1,2$ where

$$
\phi^{i}\left(\tilde{c}^{i}\right)=W^{i}\left(\tilde{c}^{i}, 1, g^{\gamma_{i}} \phi^{i}\left(\tilde{c}^{i}\right)\right)
$$

is the utility of agent $i$, weighted by $g^{\gamma_{i} t}$. Since $\beta\left(\tilde{c}^{i}, g\right)$ is not homogeneous of degree 0 in $\tilde{c}$, the rate of time preference may be different along growth paths for which consumption grows at the same rate $g$.

\section{Equilibria in a Two-Agent Economy}

In this section we apply our results to the equilibrium of a two-household economy in which each household has preferences of the kind described by Equation (8). Rather than study the competitive equilibrium directly, we appeal to the equivalence of the competitive equilibrium to the following social planners problem;

$$
\max _{C^{1}, C^{2}} u=\mu u^{1}\left(C^{1}, A\right)+(1-\mu) u^{2}\left(C^{2}, A\right)
$$

subject to the constraints

$$
\begin{aligned}
k_{t+1} & =f\left(k_{t}, g^{t}\right)-c_{t}^{1}-c_{t}^{2}, \quad t=1,2, \ldots \\
k_{0} & =\bar{k}
\end{aligned}
$$


where the technology satisfies properties $P 1-P 6$ and where $\mu$ represents the weight of agent 1 in social welfare. ${ }^{5}$ We will be concerned with the question: When is the solution to this problem a balanced growth path?

We first note that the first order conditions to the planners problem are represented by the following equations

$$
\begin{gathered}
\mu \frac{\partial u^{1}\left(C^{1}, A\right)}{\partial c_{t}^{1}}=(1-\mu) \frac{\partial u^{2}\left(C^{2}, A\right)}{\partial c_{t}^{2}} \quad t=1,2 \ldots \\
\frac{\partial u^{1}\left(C^{1}, A\right)}{\partial c_{t}^{1}}=\frac{\partial u^{1}\left(C^{1}, A\right)}{\partial c_{t+1}^{1}} \frac{\partial f\left(k_{t+1}, g^{t+1}\right)}{\partial k_{t+1}} \quad t=1,2 \ldots \\
k_{t+1}=f\left(k_{t}, g^{t}\right)-c_{t}^{1}-c_{t}^{2}, \quad t=1,2, \ldots \\
k_{0}=\bar{k}, \\
\lim _{T \rightarrow \infty}\left[\frac{\partial u^{1}\left(C^{1}, A\right)}{\partial c_{T}^{1}}\right] k_{T}=0 .
\end{gathered}
$$

We now introduce an assumption that allows us to demonstrate the consistency of balanced growth with an endogenous income distribution in the sense of Lucas and Stokey in a two agent economy.

Definition 3 (Time Preference Variation) There exist open intervals $\left(c_{a}^{1}, c_{b}^{1}\right)$ and $\left(c_{a}^{2}, c_{b}^{2}\right)$ such that for every $c^{1} \in\left(c_{a}^{1}, c_{b}^{1}\right)$ there is a $c^{2}\left(c^{1}\right) \in\left(c_{a}^{2}, c_{b}^{2}\right)$ with the property that

$$
g^{\gamma_{2}-1} \beta^{2}\left(c^{2}\left(c^{1}\right), g\right)=g^{\gamma_{1}-1} \beta^{1}\left(c^{1}, g\right)
$$

Define $k\left(c^{1}, g\right)$ by the condition

$$
f_{k}\left(k\left(c^{1}, g\right)\right)=\frac{1}{g^{\gamma_{1}-1} \beta\left(c^{1}, g\right)}
$$

\footnotetext{
${ }^{5}$ For every value of $\mu$, it follows from the second welfare theorem, that there exists a competitive economy with an initial allocation of capital, $\left\{\bar{k}^{1}(\mu), \bar{k}^{2}(\mu)\right\}$ where

$$
\bar{k}=k^{1}(\mu)+k^{2}(\mu)
$$

such that the competitive equilibrium associated with this allocation is equal to the solution to the associated planning optimum. Becker and Boyd [2] discuss a number of applications of this idea to recursive competitive economies.
} 
and let

$$
c\left(c^{1}\right)=c^{1}+c^{2}\left(c^{1}\right),
$$

represent aggregate consumption. Define the function

$$
H\left(c^{1}\right)=f\left(k\left(c^{1}, g\right)\right)-k\left(c^{1}, g\right)-c\left(c^{1}\right) .
$$

Then one of the following conditions holds: either

$$
H\left(c_{a}^{1}\right)<0, \quad H\left(c_{b}^{1}\right)>0
$$

or

$$
H\left(c_{a}^{1}\right)>0, \quad H\left(c_{b}^{1}\right)<0
$$

Time preference variation is a sufficient condition to prove existence of a balanced growth equilibrium for certain initial conditions. It states that rates of time preference along the balanced growth path must vary sufficiently with consumption such that there exist consumption sequences for which the discount rates of different agents are equated. That is the role of Equation (20). The subsidiary conditions, (22) and (23) guarantee that we can find at least one such sequence which is feasible and which satisfies the optimality conditions for an efficient production plan, (Equation (17)). The following theorem illustrates that if Definition 3 holds, there will exist a social planning problem for which the solution is a balanced growth path.

Theorem 3 Consider a two-agent economy in which the technology satisfies condition P1-P6 and the preferences of each agent are constructed from two different aggregator functions $W^{i}(c, a, u), i=$ 1,2, that satisfy assumptions $U 1-U 6$ and satisfy the homogeneity conditions of theorem 1 . Assume further that preferences satisfy Definition 3 (time preference variation). Then there exists an initial value $k_{0}$ and a welfare weight $\mu$ such that the solution to the social planning problem is a balanced growth path $\left\{k, y, c^{1}, c^{2}\right\}$.

Proof. We first show that the first-order conditions (15-19) are consistent with the existence of a balanced growth path. A balanced growth path is defined by the numbers $\left\{k, y, c^{1}, c^{2}\right\}$ where 
$k_{t}=g^{t} k, y_{t}=g^{t} y, c_{t}^{1}=g^{t} c^{1}$, and $c_{t}^{2}=g^{t} c^{2}$. From the homogeneity of utility in $C$ and $A$, there exist numbers $u^{1}$ and $u^{2}$ such that $u_{t}^{1}=g^{\gamma_{1} t} u^{1}$ and $u_{t}^{2}=g^{\gamma_{2} t} u^{2}$. Define the functions $\phi^{1}\left(c^{1}\right)$ and $\phi^{2}\left(c^{2}\right)$ as follows,

$$
\phi^{i}\left(c^{i}\right)=W^{i}\left(c^{i}, 1, g^{\gamma_{i}} \phi^{i}\left(c^{i}\right)\right), \quad i=1,2
$$

The derivative of agent $i^{\prime} s$ utility with respect to consumption at date $t$ is given by the expression

$$
\frac{\partial u^{i}\left(C^{i}, A\right)}{\partial c_{t}^{i}}=\frac{\partial W^{i}\left(c_{1}^{i}, a_{1}, u_{2}^{i}\right)}{\partial u_{2}^{i}} \ldots \frac{\partial W^{i}\left(c_{t-1}^{i}, a_{1}, u_{t}^{i}\right)}{\partial u_{t}^{i}} \frac{\partial W^{i}\left(c_{t}^{i}, a_{1}, u_{t+1}^{i}\right)}{\partial c_{t}^{i}} .
$$

Exploiting the homogeneity of $W^{i}$, we can write this expression, along a balanced growth path, as

$$
\frac{\partial u^{i}\left(C^{i}, A\right)}{\partial c^{i}}=\frac{\partial W^{i}\left(c^{i}, 1, g^{\gamma_{i}} \phi\left(c^{i}\right)\right)}{\partial u^{i}} \ldots \frac{\partial W^{i}\left(c^{i}, 1, g^{\gamma_{i}} \phi\left(c^{i}\right)\right)}{\partial u^{i}} \frac{\partial W^{i}\left(c^{i}, 1, g^{\gamma_{i}} \phi\left(c^{i}\right)\right) g^{\gamma_{i}-1}}{\partial c^{i}} .
$$

From the first order conditions of the Social Planner,

$$
\mu \frac{\partial u^{1}\left(C^{1}, A\right)}{\partial c_{t}^{1}}=(1-\mu) \frac{\partial u^{2}\left(C^{2}, A\right)}{\partial c_{t}^{2}}, \text { for all } t
$$

If we take the ratio of this condition at two adjacent dates and make use of Equation (24) we obtain the condition

$$
g^{\gamma_{1}-1} \beta^{1}\left(c^{1}, g\right)=g^{\gamma_{2}-1} \beta^{2}\left(c^{2}, g\right)
$$

where $\beta^{i}\left(c^{i}, g\right)$ is defined as

$$
\beta^{i}\left(c^{i}, g\right) \equiv \frac{\partial W^{i}\left(c^{i}, 1, g^{\gamma} \phi\left(c^{i}\right)\right)}{\partial u^{i}} .
$$

Choose $c^{1} \in\left(c_{a}^{1}, c_{b}^{1}\right)$ and find $c^{2}\left(c^{1}\right)$ such that (25) holds. The existence of the function $c^{2}\left(c^{1}\right)$ follows from Definition (3). Define the welfare weight $\mu\left(c^{1}\right)$ by the expression

$$
\mu\left(c^{1}\right) W_{c}^{1}\left(c^{1}, 1, g^{\gamma} \phi^{1}\left(c^{1}\right)\right)=\left(1-\mu\left(c^{1}\right)\right) W_{c}^{2}\left(c^{2}\left(c^{1}\right), 1, g^{\gamma} \phi^{1}\left(c^{2}\left(c^{1}\right)\right)\right)
$$

and define $k\left(c^{1}, g\right)$ to be the unique solution to the Equation

$$
f_{k}\left(k\left(c^{1}, g\right)\right)=\frac{1}{g^{\gamma_{1}-1} \beta^{1}\left(c^{1}, g\right)} .
$$

The existence of the function $k\left(c^{1}, g\right)$ for all $c^{1} \in\left(c_{a}^{1}, c_{b}^{1}\right)$ is implied by the Inada conditions, (assumptions $P 5$ and $P 6$ ). It remains to show that we can find a $c^{1}$ that is feasible: but this is 
guaranteed by $(22)$ and $(23)$ since the function $H\left(c^{1}\right)$ is continuous and has at least one zero. We have demonstrated that our utility function is consistent with the first order conditions (15-17). Consistency with the transversality condition, Equation (19) follows from U5 which bounds the discount factor and ensures that marginal utilities grow more slowly than the growth rate of the economy.

Theorem 3 does not assert that the balanced growth path is consistent with equilibrium for all welfare weights. For this to be true, one would require something analogous to the Inada conditions applied to preferences. It asserts instead that if discount rates vary enough with consumption then there exist welfare weights that are consistent with existence of a balanced growth path. The implication for a decentralized equilibrium is that there exists some initial wealth distribution that is consistent with the range of variation in discount factors permitted by Definition 3, for which an equilibrium exists.

The theorem also says nothing about convergence to the balanced growth path. If the initial capital stock happens to exactly equal the balanced growth stock $k$, then the solution to the social planner's problem will place the economy immediately on its balanced growth path. If the initial stock is not equal to $k$, the economy may or may not converge to the balanced growth path. In the case of an exchange economy, Lucas and Stokey give a two-person example in which a non-inferiority condition plus the assumption of increasing marginal impatience are sufficient to guarantee local convergence to balanced growth. In the following section we apply the Lucas-Stokey results to our economy.

\section{Two Examples}

\subsection{The Lucas Stokey Example}

We begin with a straightforward application of our main theorem to the exchange economy described in Section 8 of Lucas and Stokey $([11])$. We amend their example by assuming that the endowment of the economy is growing with exogenous growth factor, $g>1$. In our economy, utility 
functions $U^{i}\left(C^{i}, A\right)$ are constructed from homogenous aggregators

$$
W^{i}(c, a, u)
$$

that satisfy assumptions $U_{1}-U_{6}$. We assume that $A=\left\{g, g^{2}, g^{3} \ldots\right\}$ is an exogenous sequence of growth factors and we define weighted consumption sequences $\tilde{C}^{i}$ as follows

$$
\tilde{C}^{i}=\left\{\frac{c_{1}^{i}}{1}, \frac{c_{2}^{i}}{g}, \ldots\right\}
$$

We assume further that utility is homogenous of degree $\gamma_{i}$ in $C^{i}$ and $A$ and hence we can define an aggregator $\tilde{W}^{i}$

$$
\tilde{W}^{i}\left(\tilde{c}_{t}^{i}, \tilde{u}_{t+1}^{i}\right)=W^{i}\left(\frac{c_{t}^{i}}{g^{t}}, 1, g^{\gamma_{i}} \frac{u_{t+1}^{i}}{g^{(t+1) \gamma_{i}}}\right)=\frac{W\left(c_{t}^{i}, a_{t}, u_{t+1}^{i}\right)}{g^{\gamma_{i}}}, \quad i=1,2
$$

such that

$$
\tilde{u}_{t}=\tilde{W}^{i}\left(\tilde{c}_{t}^{i}, \tilde{u}_{t+1}\right)
$$

The Social Planning problem for the transformed economy can be written in the same form as the problem studied in Section 5 of Lucas and Stokey [11]. The weighted allocations $\left\{\tilde{c}_{t}^{i}\right\}$ are stationary by construction and the weighted aggregators $\tilde{W}^{i}\left(\tilde{c}_{t}^{i}, \tilde{u}_{t+1}^{i}\right)$ satisfy assumptions $W_{1}-W_{5}$ (Lucas and Stokey page 142). Assumptions $P_{1}-P_{6}$ imply that the technology is regular in the sense of Burmeister [5]. For aggregators in this class, Lucas and Stokey prove the existence of a unique stationary state (Theorem 4 on page 162). For a two person exchange economy that satisfies two additional conditions they prove that the equilibrium is locally stable (Theorem 5) and they demonstrate that the wealth distribution associated with the steady state is non-degenerate.

The first condition required for the Lucas Stokey theorem is that $c$ and $u$ are noninferior 'goods' for both consumers; that is,

$$
c<c^{\prime} \text { and } u>u^{\prime} \Longrightarrow \frac{\tilde{W}_{c}^{i}(c, u)}{\tilde{W}_{u}^{i}(c, u)}>\frac{\tilde{W}_{c}^{i}\left(c^{\prime}, u^{\prime}\right)}{\tilde{W}_{u}^{i}\left(c^{\prime}, u^{\prime}\right)}, i=1,2 .
$$

This condition is relatively innocuous. The more restrictive condition is the assumption of increasing marginal impatience, that is;

$$
\tilde{W}_{u}^{i}(c, \phi(c))
$$


is decreasing in $c$. It is precisely this condition that breaks down in the case of homogenous aggregators when $A$ does not directly enter preferences since we have shown in Theorem 2 that, in this case, the rate of time preference is independent of consumption.

$$
\beta^{i}(c, g)=\tilde{W}_{u}^{i}\left(c, g^{\gamma} \phi(c)\right)=\beta^{i}(g)
$$

By introducing $A$ into preferences we are able to maintain homogeneity (required for balanced growth) and construct examples of utility functions that satisfy the Lucas Stokey assumptions and that are, therefore, consistent with the existence of non-trivial economies in which the equilibrium wealth distribution is non-degenerate and is obtained as the stable fixed point of a competitive equilibrium.

\subsection{A Small Open Economy Example}

Our second example generalizes the case of a small open economy in which the representative agent has preferences of the form

$$
u_{t}=\sum_{t=1}^{\infty} \beta^{t-1} \frac{\left(c_{t}\right)^{\theta}}{\theta} .
$$

We assume that the agent may borrow or lend at constant gross interest rate $R$ and that the agent has an endowment stream

$$
w_{t}=g^{t} w
$$

In this case, it is well known that, for a constant $\beta$ and $R$, the dynamics of this economy are characterized by a unit root. The asymptotic debt of the representative country depends on initial conditions. Moreover, the existence of a stationary equilibrium for the small open economy depends on the knife-edge property that the exogenous rate of time-preference of the individual country should, fortuitously, be equal to the exogenous world real rate of interest; that is, $\beta R=1$.

Non-separable preferences, similar to the ones we study in this paper have been used by Mendoza ([12]) and Schmitt-Grohé and Uribe [15], to close models of a small open economy. Our work implies that existing formulations of recursive preferences, such as those of Uzawa [16], Epstein-Hynes [7] 
or Lucas-Stokey [11], are inconsistent with growing economies. We show how to extend these studies to growing economies.

We propose the following specification. Let world wealth be given by the expression

$$
a_{t}=g^{t} a
$$

and let the individual's preferences be constructed from the aggregator function

$$
u_{t}=W\left(c_{t}, a_{t}, u_{t+1}\right) \equiv \frac{(1-\beta) c_{t}^{\theta} a_{t}^{1-\theta}}{\theta}+\frac{\beta u_{t+1}^{\delta} a_{t}^{1-\delta}}{\delta}
$$

where $W$ satisfies assumptions $U 1-U 6$. We also assume $0<\delta<1, \quad \theta<1 .^{6}$ Note that this aggregator is linearly homogenous and, when $\delta=1$, it generates the constant relative risk aversion preferences of Equation (29).

The individual faces the sequence of budget constraints

$$
\begin{aligned}
& b_{t}=b_{t-1} R+w_{t}-c_{t}, \quad t=1, \ldots \\
& b_{0}=\bar{b}_{0}
\end{aligned}
$$

where $b_{t}$ is domestic assets at date $t, w_{t}$ is exogenous wage income defined by the sequence $w_{t}=g^{t} w$, and $R$ is the exogenous market interest factor.

The utility maximizing individual chooses a consumption sequence that equates his marginal rate of substitution to the world interest factor $R$;

$$
\left(\frac{c_{t}}{a_{t}}\right)^{\theta-1}=\beta\left(\frac{c_{t+1}}{a_{t+1}}\right)^{\theta-1}\left(\frac{u_{t+1}}{a_{t+1}}\right)^{\delta-1} R g^{\delta-1} .
$$

Equations (30) that defines recursive preferences and (31) that represents the first-order condition for maximization of utility can be expressed as a system of two difference equations in the transformed variables $\tilde{u}_{t}=u_{t} / a_{t}$ and $\tilde{c}_{t}=c_{t} / a_{t}$;

$$
\begin{aligned}
\tilde{u}_{t} & =\frac{(1-\beta) \tilde{c}_{t}^{\theta}}{\theta}+\frac{\beta g^{\delta} \tilde{u}_{t+1}^{\delta}}{\delta}, \\
\tilde{c}_{t}^{\theta-1} & =\beta R g^{\delta-1} \tilde{c}_{t+1}^{\theta-1} \tilde{u}_{t+1}^{\delta-1} .
\end{aligned}
$$

\footnotetext{
${ }^{6}$ Since the composition of concave functiuons is concave, concavity of the aggregator $W\left(c, u^{\prime}\right)$ in $c$ and $u^{\prime}$ is a sufficient condition for $u(C)$ to be concave in $C$. This is guaranteed by the assumptions, $\theta<1, \quad \delta<1$.
} 
Let $\tilde{b}_{t}$ be the ratio of net assets to world wealth, that is, $\tilde{b}_{t}=b_{t} / a_{t}$. The budget constraint, in transformed variables, takes the form;

$$
\begin{aligned}
& \tilde{b}_{t}=\tilde{b}_{t-1} \frac{R}{g}+\tilde{w}-\tilde{c}_{t}, \quad t=1, \ldots \\
& \tilde{b}_{0}=\tilde{b}_{0},
\end{aligned}
$$

where $\tilde{w}_{t}=w_{t} / g^{t}$. Equations (32-34) represent a system of three difference equations in three variables, $\tilde{u}_{t}, \tilde{c}_{t}, \tilde{b}_{t}$ with a single initial condition, $\tilde{b}_{0}$. The unique balanced growth path is defined by the equations

$$
\bar{u}=\left(\frac{1}{\beta R}\right)^{\frac{1}{\delta-1}} \frac{1}{g}, \quad \bar{c}=\left[\frac{\theta}{1-\beta}\left(\bar{u}-\frac{\beta}{\delta} g^{\delta} \bar{u}^{\delta}\right)\right]^{\frac{1}{\theta}}, \quad \bar{b}=\frac{(\bar{c}-\tilde{w})}{R / g-1} .
$$

In the appendix we show that the system (32-34), when linearized around the balanced growth path, has one root below 1 and one root above 1 . We also assume that $R>g$ which is a necessary condition for the wealth of the agent to be well defined. Under these assumptions, the subsystem (32-33) defines a linear function

$$
d \tilde{u}_{t}=\mu_{1} d \tilde{c}_{t}
$$

and a scalar difference equation

$$
d \tilde{c}_{t+1}=\mu_{2} d \tilde{c}_{t}
$$

where $0<\mu_{2}<1$ and $d \tilde{x}$ is the percentage deviation of $\tilde{x}$ from $\bar{x}$. This subsystem converges back to the balanced growth path for $\tilde{c}_{t}$ in the neighborhood of $\bar{c}$. The initial value of this difference equation, $\tilde{c}_{0}$, is chosen to satisfy the transversality condition, that is, to ensure that

$$
\tilde{b}_{0}+\sum_{t=1}^{\infty} Q_{0}^{t}\left(w_{t}-\tilde{c}_{t}\right)=0
$$

where

$$
Q_{0}^{t}=\frac{1}{(R / g)^{t-1}}
$$

Agents in our example display an endogenous discount rate and their long-run position in the world income distribution is a function of their preferences. 


\section{Conclusion}

In this paper we have studied the circumstances under which one can model an endogenous income distribution in a growing economy. We have shown that the homogeneity of the welfare aggregator (a property that is required for balanced growth) has strong implications in multiple agent environments. In general, balanced growth equilibria do not exist in a multi-agent economy except for the special case where all agents have the same constant rate of time preference. This case is uninteresting since it eliminates meaningful preference heterogeneity which is one of the key motivations for studying recursive preferences to begin with.

Given that balanced growth provides a fairly good description of the industrialized economies, these findings highlight a problematic feature of recursive preferences. We have suggested a generalization of the recursive preference structure that permits the coexistence of balanced growth equilibria with multiple agent economies and recursive preferences. Our extension requires preferences to be explicitly time dependent and the aggregator to be homogeneous in current consumption, future utility and an exogenous (time dependent) growth factor. 


\section{Appendix}

The Roots of $J$. In our example the system can be linearized around the balanced growth path state. The linearized system has the form

$$
\left[\begin{array}{l}
d \tilde{c}_{t+1} \\
d \tilde{u}_{t+1}
\end{array}\right]=J\left[\begin{array}{l}
d \tilde{c}_{t} \\
d \tilde{u}_{t}
\end{array}\right]
$$

where

$$
J=\left[\begin{array}{cc}
\frac{(1-\delta) s \theta}{(1-\theta)(1-s) \delta}+1 & \frac{-(1-\delta)}{(1-\theta)(1-s) \delta} \\
\frac{-s \theta}{(1-s) \delta} & \frac{1}{(1-s) \delta}
\end{array}\right] .
$$

We are interested in the roots of $J$ : To study their properties, consider the following Lemma.

Lemma 2 Let

$$
f(X, \mu)=X^{2}-(T+\mu) X+D
$$

be a polynomial in $X$ with roots $\lambda_{1}(\mu)$ and $\lambda_{2}(\mu)$. Suppose further that when $\mu=0$, both roots are real and $\lambda_{1}(0)<\lambda_{2}(0)$. Since the discriminant $\left((T+\mu)^{2}-4 D\right)$ is increasing in $\mu$, it follows that

$$
\lambda_{1}(\mu)<\lambda_{1}(0), \quad \lambda_{2}(\mu)>\lambda_{2}(0)
$$

for all $\mu>0$.

In our example, the determinant of $J$ is given by

$$
D=\frac{1}{(1-s) \delta}
$$

and the sum of the roots, (the Trace of $J$ ) is:

$$
T=\frac{1}{(1-s) \delta}+1+\mu(\delta)
$$

where

$$
\mu(\delta)=\frac{s \theta(1-\delta)}{\delta(1-s)(1-\theta)}
$$

For $\delta=1$ the roots of the characteristic polynomial are given by $\lambda_{1}=1$ and $\left.\lambda_{2}=1 /(1-s) \delta\right)>1$. When $\delta<1 \mu(\delta)>0$. Hence, for values of $\delta<1$, in the neighborhood of $\delta=1, \mu(\delta)>0$ and, by 
lemma $2 \lambda_{1}(\delta)<1$ and $\lambda_{2}(\delta)>1$. It follows that, provided $\delta$ is not too far from 1 , (and hence the smaller root is not less than -1 ) the roots of the characteristic polynomial split around 1 in absolute value. 


\section{References}

[1] Becker, Robert, (1980). "On the Long-Run Steady States in a Simple Dynamic Model of Equilibrium with Heterogeneous Households", Quarterly Journal of Economics, 95, (2), 375382.

[2] Becker, Robert A. and John H. Boyd III, (1997). Capital Theory, Equilibrium Analysis and Recursive Utility, Blackwell Publishers.

[3] Ben-Gad, Michael, (1999). "Balanced-Growth -Consistent Recursive Utility and Heterogenous Agents", Journal of Economic Dynamics and Control 23, pp 459-462.

[4] Boyd, John H. III, (1990). "Recursive Utility and the Ramsey Problem". Journal of Economic Theory, 50, 326-345.

[5] Burmeister, Edwin. "Capital Theory and Dynamics", Cambridge University Press London/New York 1980.

[6] Dolmas, Jim, 1996, "Balanced-Growth-Consistent Recursive Utility," Journal of Economic Dynamics and Control 20, 657-680.

[7] Epstein, Larry G. and J. Alan Hynes, (1983). "The Rate of Time Preference and Dynamic Economic Analysis", Journal of Political Economy, 91, 611-635.

[8] Kaldor, Nicholas, (1961). "Capital Accumulation and Economic Growth," in Lutz, F. A., and Hague, D.C. The Theory of Capital. New York, St. Martin's Press.

[9] King, Robert, Charles Plosser and Sergio Rebelo, (1988). "Production Growth and Business Cycles: Technical Appendix", mimeo.

[10] Koopmans, Tjalling C., (1960). "Stationary Ordinal Utility and Impatience," Econometrica, 28, 287-309. 
[11] Lucas, Robert E. Jr. and Nancy L. Stokey, (1984). "Optimal Growth with Many Consumers". Journal of Economic Theory, 32, 139-171.

[12] Mendoza, Enrique G, (1991). "Real Business Cycles in a Small Open Economy", American Economic Review, 81:4, pp. 797-818.

[13] Ramsey, Frank, (1928). "A Mathematical Theory of Saving," Economic Journal 38, pp 543-59.

[14] Rebelo, Sergio, 1991. "Long-Run Policy Analysis and Long-Run Growth," Journal of Political Economy 99, 500-521.

[15] Schmitt-Grohé, Stephanie and Martin Uribe (2003). "Closing Small Open Economy Models", Journal of International Economics 61, pp. 163-185.

[16] Uzawa, H, (1968). "Time Preference, the Consumption Function, and Optimum Asset Holdings"., in Value Capital and Growth: Papers in Honour of Sir John Hicks" (J.N. Wolfe, Ed.), Edinburgh University Press, Edinburgh. 